\section{Correspondence on 'EULAR recommendations for the management of antiphospholipid syndrome in adults'}

2019 antiphospholipid syndrome (APS) guideline ${ }^{1}$ has been launched and has given recommendations to the rheumatologists on how to manage APS in different situations. The guideline has been presented in detail but there are still some questions remain to be discussed. For example, we are still confused in some circumstances, in secondary APS, such as systemic lupus erythematous, glucocorticoids (GC) is widely used. But according to the guideline in primary APS, GC treatment is recommended only in first trimester of pregnancy or in catastrophic APS (CAPS). But what about in second and third trimester of pregnancy? What dosage of GC is recommended? It needs more open discussion.

In terms of CAPS, the definition has a strict request on biopsy. Yet, in the clinic practice, there are little chance to do the biopsy. Besides, thrombosis happened in 1 week is also a criterion hard to meet; sometimes, it could happen in more than 1 week. Is it possible that in the future, the definition of CAPS will be modified? Although we could give the diagnose of probable CAPS in these cases, patients are still in danger and have a great risk of severe complications, whose treatment should be similar to CAPS. For instance, GC and plasma exchange need to be used. Furthermore, the guideline did not recommend the choice of disease-modifying antirheumatic drugs in severe cases, is there a preference? More studies are needed to answer this question as well.

Another circumstance is that patients with high-risk aPL profiles and new-onset thrombosis, besides heparin, is there a high-priority for GC and plasma exchange or intravenous immunoglobulins ? Moreover, in obstetric APS, another argument is that if a woman with positive medium-high titres of antiphospholipid antibodies, but with just once or twice spontaneous miscarriages (less than 10 weeks' gestation), meaning not meeting the APS classification criteria, what should be done, to use aspirin or not? For these older pregnant women, it sounds like a disaster to have another miscarriage without any intervention. Maybe these questions need more evidence and more clear explanation from experts.
Zhuochao Zhou, Fan Wang, Yue Sun, Jialin Teng $\odot$, Honglei Liu, Xiaobing Cheng, Yutong Su $\odot$, Hui Shi $\odot$, Yijun You, Chengde Yang $\odot$, Junna Ye

Correspondence to Dr Junna Ye, Department of Rheumatology and Immunology, Ruijin Hospital, Shanghai Jiao Tong University School of Medicine, Shanghai, China; yjn0912@qq.comMr Chengde Yang; yangchengde@sina.com

Contributors Concept and writing: JY, CY, ZZ and FW. Revising: YS, JT, H-LL, XC, YS, $\mathrm{HS}$ and YY.

Funding This work was supported by the National Natural Science Foundation of China (No. 81801592)

Competing interests None declared.

Patient and public involvement Patients and/or the public were not involved in the design, or conduct, or reporting, or dissemination plans of this research.

Patient consent for publication Not required.

Ethics approval This study was approved by the Ethics Committee of Ruijin Hospital.

Provenance and peer review Not commissioned; internally peer reviewed.

(c) Author(s) (or their employer(s)) 2020. No commercial re-use. See rights and permissions. Published by BMJ.

ZZ and FW contributed equally.

D) Check for updates

To cite Zhou Z, Wang F, Sun Y, et al. Ann Rheum Dis Epub ahead of print: [please include Day Month Year]. doi:10.1136/annrheumdis-2020-218950

Received 31 August 2020

Accepted 2 September 2020

\section{S Linked}

http://dx.doi.org/10.1136/annrheumdis-2020-219048

Ann Rheum Dis 2020;0:1. doi:10.1136/annrheumdis-2020-218950

\section{ORCID iDs}

Jialin Teng http://orcid.org/0000-0003-1016-9064

Yutong Su http://orcid.org/0000-0003-0488-2939

Hui Shi http://orcid.org/0000-0002-3574-8807

Chengde Yang http://orcid.org/0000-0002-3720-634X

Junna Ye http://orcid.org/0000-0002-2823-4349

\section{REFERENCE}

1 Tektonidou MG, Andreoli L, Limper M, et al. EULAR recommendations for the management of antiphospholipid syndrome in adults. Ann Rheum Dis 2019;78:1296-304. 